\title{
Minimizing uncertainty in complete automation of Douglas-Peucker Algorithm for geospatial mapping
}

Rong Wang

Lanzhou Jiaotong University

Muhammad Shafeeque ( $\nabla$ shafeequ@uni-bremen.de)

University of Bremen: Universitat Bremen https://orcid.org/0000-0002-2152-6672

Haowen Yan

Lanzhou Jiaotong University

Lu Xiaoming

Lanzhou Jiaotong University

\section{Research Article}

Keywords: Optimal distance threshold, Map scale change, Automatic map generalization, Geometric accuracy, Spatial similarity relationships

Posted Date: December 29th, 2021

DOI: https://doi.org/10.21203/rs.3.rs-1162837/v1

License: (1) (1) This work is licensed under a Creative Commons Attribution 4.0 International License. Read Full License 
1 Minimizing uncertainty in complete automation of Douglas-Peucker

$2 \quad$ Algorithm for geospatial mapping

\section{Abstract}

4 It is qualitatively evident that the greater the map scale change, the greater the optimal

5 distance threshold of the Douglas-Peucker Algorithm, which is used in polyline

6 simplification. However, no specific quantitative relationships between them are known

7 by far, causing uncertainties in complete automation of the algorithm. To fill this gap,

8 the current paper constructs quantitative relationships based on the spatial similarity

9 theories of polylines. A quantitative spatial similarity relationship model was proposed

10 and evaluated by setting two groups of control experiments and taking $<\mathrm{C}, \mathrm{T}>$ as

11 coordinates. In order to realize the automatic generalization of the polyline, we verified

12 whether these quantitative relationships could be fitted using the same function with

13 the same coefficients. The experiments revealed that the unary quadratic function is the

14 best, whether the polylines were derived from different or the same geographical feature

15 area(s). The results also show that using the same optimal distance threshold is

16 unreasonable to simplify all polylines from different geographical feature areas. On the

17 other hand, the same geographical feature area polylines could be simplified using the

18 same optimal distance threshold. The uncertainties were assessed by evaluating the

19 automated generalization results for position and geometric accuracy perspectives using

20 polylines from the same geographic feature areas. It is demonstrated that in addition to

21 maintaining the geographical features, the proposed model maintains the shape 
characteristics of polylines. Limiting the uncertainties would support the realization of completely automatic generalization of polylines and the construction of vector map geodatabases.

Keywords: Optimal distance threshold; Map scale change; Automatic map generalization; Geometric accuracy; Spatial similarity relationships

\section{Introduction}

In realistic cartography, different cartographers always select different maps as the generalization results, and in most situations, cartographers often need to input parameter(s) in the execution of an algorithm. This humans' constant interference consequently leads to the non-automation of generalized algorithms, which decreases the efficiency of map generalization and increases the uncertainty of the resulting maps (Yan 2014; Makris et al., 2021). Linear data such as contour, road network, and rivers are the main spatial data types widely used in different fields. In the process of downsizing small-scale maps or constructing vector map databases, it is indispensable to generalize polylines from one larger scale (e.g., 1:5000) to corresponding generalized counterparts at the other smaller scale (e.g., 1:50000). Therefore, it is extremely essential to realize the completely automatic generalization of polylines.

Numerous scholars have been making tremendous efforts to investigate the realization of automatic polyline generalization issues (Ai et al. 2016; Shen et al. 2018; Kronenfeld et al. 2020). Meanwhile, various methods have been presented (Mcmaster 1987), such 
as the Douglas-Peucker algorithm (DP) (Douglas and Peucker 1973), Li-Openshaw (Li and Openshaw 1992), Bend Group algorithm (Qian HZ 2017). However, the problems are only partially solved. Among these approaches, the DP remains the most wellknown and effective polyline simplification algorithm in map generalization (Ramer 1972; Hershberger and Snoeyink 1992; Saalfeld 1999; Yan 2014; Sandu et al., 2015). The evaluation and minimization of uncertainties in polyline simplification methods might validate these algorithms' acceptability, consistency, and advanced application for automatization.

Spatial similarity relationships are critically important measures for the accuracy assessment of map generalization results (Yan 2015, 2016; Makris et al., 2021), which have attracted the increasing attention of cartographers in recent years. However, few studies have been published on the automatic generalization of polylines based on spatial similarity relationships. The reason mainly focuses on three aspects: Firstly, no in-depth investigations on integrating spatial similarity degree into map automatic generalization are available. Only a few studies have been tested (Yan 2014), i.e., it is ambiguous about how to automatically calculate the transition conditions threshold of DP using the spatial similarity degree. As a result, it leads to constant human intervention in the execution of DP. Secondly, very little is known about the specific quantitative functional relationship between optimal distance threshold and map scale change. Is it linear or nonlinear? Is it a linear equation with one variable or a quadratic equation, or is it a logarithmic function? These questions are still being sought. Thirdly, 
63 when referring to the effect of regional geographical features on selecting distance threshold, some cartographers believe that it should be considered, while others argue that there is no need (Li and Sui 2000). Besides, they assume that the reason why the map is generalized is only the change of map scale, while other surrounding factors remain unchanged. For example, regardless of the chosen threshold, the degree of terrain fragmentation remains unchanged before and after the generalization. Hence, the conflicting opinions adversely influence the determination of the optimal distance threshold and cause uncertainty. A computational model based on quantitative spatial

71 similarity relationships might overcome the challenge of realizing an entirely automatic generalization of the polylines.

73 Constructing the quantitative functional relationships between the optimal distance

74 threshold (T) and map scale change (C) would realize the completely automatic 75 generalization of polylines based on the DP without manual parameterization. 76 Meanwhile, the optimal distance threshold mainly depends on the magnitude of map 77 scale change. Technically, when the spatial similarity degree between the intermediate 78 polyline $\left(\mathrm{L}_{\mathrm{i}}\right)$ simplified by DP and the standard target scale polyline (LM2) reaches the 79 maximum, the corresponding threshold is obviously the optimal distance threshold. The 80 main goal of the current research is to minimize the uncertainties in determining the 81 optimal distance threshold for improving the complete automation of the DP algorithm. 


\section{Methodology}

83 In order to automatically calculate the optimal distance threshold and realize the completely automatic generalization of the individual polyline, this paper firstly constructs and validates the spatial similarity evaluation model by quantitatively selecting the evaluation index and determining its weight, and then calculates the optimal distance threshold based on the spatial similarity relations. Afterward, the optimal distance threshold and corresponding map scale change are recorded when the similarity degree between the intermediate result and the standard target scale polyline reaches the maximum. Then taking $<\mathrm{C}, \mathrm{T}>$ as the coordinates, the best quantitative

91 empirical formula is fitted using the regression analysis and least square model. The generalization results are finally validated from the position and geometric accuracy perspectives to confirm the acceptability of the introduced methods.

\section{2.1. Principle of parameter optimization of DP algorithm}

Douglas-Peucker algorithm (DP) is a well-known and effective simplification algorithm of polylines (Douglas and Peucker 1973). DP has been widely used in map generalization to reduce the number of points on a curve (Yan 2014; Gu et al. 2016).

98 According to its simplification fundamentals, we can clearly know that the degree that 99 polyline is simplified mainly depends on the threshold of each simplification process,

100 i.e., the initial threshold and step size. Moreover, in which points are deleted, the order 101 is the opposite of that when points are reserved. However, it is consistent with the 

deleted and the degree to that a polyline is generalized. Hence, the current research is

104 aimed to find an appropriate approach for determining the quantitative relationship

105 between $\mathrm{T}$ and $\mathrm{C}$. To this end, the order that points to be deleted is firstly determined

106 by reversing the order of points to be selected. As a result, the similarity between the intermediate result and the standard target scale polyline reaches the maximum; the

108 corresponding threshold $\lambda_{\mathrm{k}}$ is the optimal distance threshold that the polyline is

109 generalized from scale $M_{1}$ to scale $M_{2}$. Finally, the point pairs $(C, T)$ used to fit the 110 quantitative relations between them are recorded after multiple circulative iterations

111 (Figure 1).

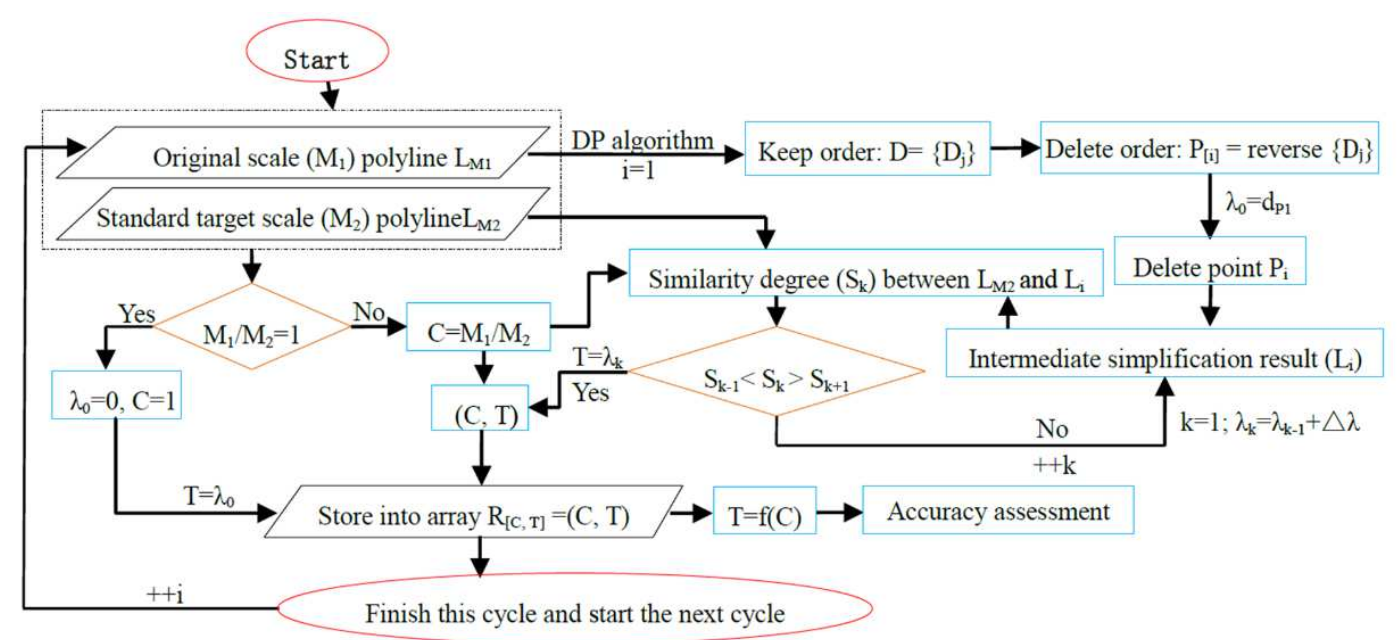

113 Figure 1. Flow chart of the methodology applied in this study. $\lambda_{0}$ is the initial distance threshold. $\Delta \lambda$ is a

114 gradually changing value, and the value of $\Delta \lambda$ is inversely correlated with the similarity degree between $L_{M 2}$

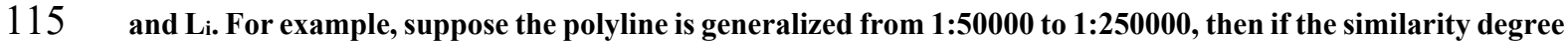

116 (S) between $L_{M 1}$ and $L_{i}$ is less than $0.6, \Delta \lambda$ will be $0.02 \mathrm{~km}$, while if $S$ is more than 0.6 and less than $0.8, \Delta \lambda$

117 will be $0.01 \mathrm{~km}$; and if $S$ is more than $0.8, \Delta \lambda$ will be $0.005 \mathrm{~km}$. 


\section{2.2. Selection of evaluation index and determination its weights}

120 The judgment of spatial similarity degree is the essence of map generalization. However,

121 people often ambiguity about the similarity between two objects when judging the spatial similarity relationship between multi-scale maps because they remain uncertain

123 about what properties should be considered in similarity assessments (Yan 2010).

124 Therefore, among many polyline properties, including length, distance, complexity,

125 sinuosity, etc., it is necessary to extract major properties that influence people's

126 judgment at first and then determine its corresponding weights to construct a spatial

127 similarity evaluation model.

128 According to previous research, 12 properties can be used to evaluate the spatial

129 similarity between multi-scale polylines, including position (Olteanu-Raimond et al.

130 2015), length (Zhang et al. 2014), distance (Deng 2007, Tong et al. 2014), size,

131 sinuosity (Danlel et al. 2014), complexity, orientation (Samal 2004, Olteanu-Raimond

132 et al. 2008), area, shape, buffer-overlapped area, degree of node and attribute. These

133 can be further divided into geometric properties and thematic attribute properties (Li et

134 al. 2006, Zhang 2009). Due to the variety of the geometric properties, it is not clear to

135 choose what combination of geometric properties to produce the best solution. In

136 addition, if all properties have been considered, correlated and redundant information

137 is likely imported, which may lead to low computational efficiency and the accuracy of 
138 the result. This phenomenon can be explained by the curse of dimensionality or

139 Hughes's effect (Hughes 1968). Using as few indicators as possible to express as much

140 polyline information as possible should be solved first. Therefore, absolute distance

141 matrix D between factors is employed to overcome the above problem and measure the

142 closeness of the indicators based on the results of genetic algorithm.

143

$$
D_{(12 \times 12)}=\left[\begin{array}{lllllllllllllllll}
0 & & & & & & & & & & & & & \\
3.22 & 0 & & & & & & & & & & \\
1.94 & 3.49 & 0 & & & & & & & & & \\
4.22 & 2.46 & 3.95 & 0 & & & & & & & & \\
5.14 & 4.41 & 4.13 & 3.56 & 0 & & & & & & & \\
1.23 & 3.39 & 1.99 & 4.51 & 5.24 & 0 & & & & & & \\
1.94 & 3.22 & 1.60 & 3.71 & 4.39 & 1.99 & 0 & & & & & \\
1.45 & 3.18 & 2.39 & 4.46 & 5.37 & 0.99 & 2.72 & 0 & & & & \\
5.04 & 3.25 & 4.46 & 2.32 & 2.73 & 5.27 & 5.49 & 5.49 & 0 & & & \\
3.16 & 2.98 & 3.73 & 4.59 & 4.60 & 3.79 & 4.79 & 3.19 & 4.79 & 0 & & \\
5.28 & 4.53 & 4.71 & 3.95 & 3.86 & 6.02 & 4.12 & 5.72 & 4.04 & 4.12 & 0 & \\
4.21 & 4.06 & 4.02 & 4.04 & 2.62 & 4.88 & 3.42 & 4.89 & 3.45 & 3.42 & 3.42 & 0
\end{array}\right]
$$

144 where, $\mathrm{R}$ is a symmetric matrix, and $d_{i j}=\sum_{k=1}^{n}\left|w_{i k}-w_{j k}\right|(i, j=1,2, \ldots, 12, k=$

$1451,2, \ldots, 9), w_{i k}$ represents the kth factor that affects the ith data group in Table 5 of

146 Chehreghan's research.

147 And then, the direct cluster genealogy diagram (Figure 2) is derived based on the matrix

148 R. 


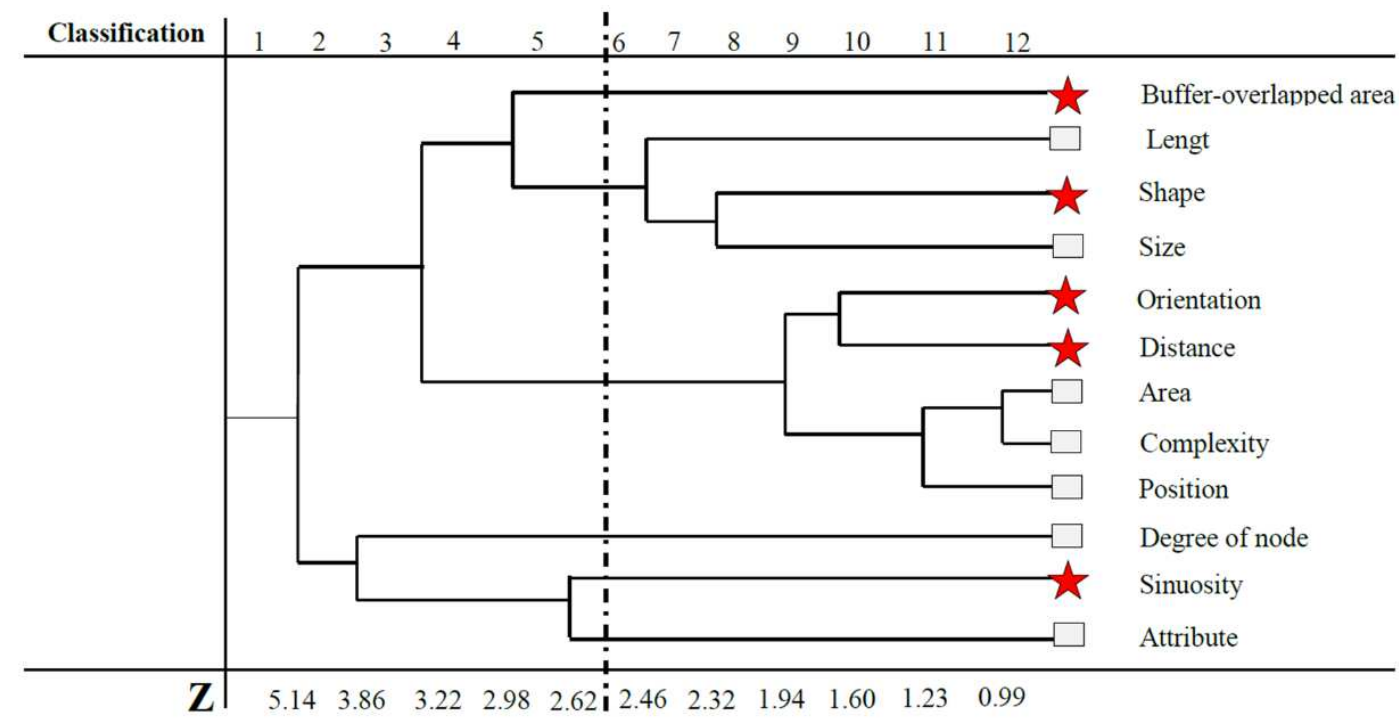

151 According to the $\mathrm{F}$ statistical analysis of cluster analysis, $\mathrm{F}>F_{\propto}\left(f_{1}, f_{2}\right)=2.494(\propto=$

$1520.05, f_{1}=r-1=5, f_{2}=n-r=3$ ), which indicates that the classes in Figure 2 are

153 significantly different, the classification strategy is reasonable. It can be determined that

$154 \mathrm{Z}$ belongs to $(2.46,2.62)$ because the attribute as the thematic property can be extracted

155 as an independent class, meanwhile, the clustering degree of other geometric properties

156 reaches maximum. Finally, the impact indices are clustered into six categories:

$157\{$ Attribute $\},\{$ Sinuosity $\},\{$ Degree of node $\},\{$ Position, Complexity, Area, Distance,

158 Orientation $\}$, \{Size, Shape, Length $\}$, \{Buffer-overlapped area $\}$. In this paper, only

159 geometric properties were considered because, in most situations, cartographers pay

160 more attention to the geometric attributes of polylines and ignore their thematic attribute

161 information (Deng 2007). It should be noted that \{degree of node\}, \{Position,

162 Complexity, Area $\}$ and $\{$ Size, Length $\}$ are excluded from the selected impact factors 
163 since they impact only a few part groups of the dataset. Finally, the chosen geometric

164 factors are $\{$ Sinuosity\}, \{Distance, Orientation\}, \{Shape $\}$ and $\{$ Buffer-overlapped

165 area\}. Except for distance and orientation, the correlation coefficient $\left|R_{x y}\right|$ between the

166 selected geometric factors is less or equal to 0.3 , which is consistent with the basis for

167 the exclusivity of index selection. Although distance and orientation were clustered in

168 one class, and their correlation coefficient $\left|R_{x y}\right|$ is 0.76 , they impact all groups of

169 datasets in stability test, which indicates that both factors are very stable. Thus,

170 Sinuosity, Distance, Orientation, Shape, and Buffer-overlapped area are chosen. It is

171 generally not allowed to move polylines on maps in the process of map generalization,

172 i.e., orientation between polylines is not changed after map generalization. Therefore,

173 Sinuosity, Distance, Shape, and Buffer-overlapped area are finally chosen as evaluation

174 indices.

175 Distance is a fundamental concept in geospatial science. According to the previous

176 research, it can be known that compared with Euclidian distance (Peuquet 1992),

177 Bottleneck distance (Efrat et al. 2001), and Fréchet distance (Nayyeri et al. 2015),

178 Hausdorff distance is one of the most used distances for spatial objects in GIS, but it is

179 sensitive to the shape of the objects, especially to the outliers. Besides, it does not satisfy

180 the change law of similarity (Li et al., 2018). Therefore, Mean- Hausdorff distance

181 (MHD) is selected as the distance similarity metric to obtain more stable and accurate

182 result (Deng et al. 2007). On the other hand, the shape is viewed as the most crucial

183 geometric factor that describes planar curves. The essence of shape similarity is to judge 
184 the coincidence degree between polylines. Therefore, $\mathrm{Li}$ (2018) comparatively assessed

185 the advantages and disadvantages of different measurement indices through

186 experiments, e.g., Vertex Reserved, Length Ratio, Turning Function, and Dual-side

187 Bend Forest Shape Similarity. The results indicated that the cumulative turning function

188 (Fan et al. 2014) (Equation (1)) could eliminate the interference of detail difference,

189 which is more suitable for the comparison between multi-scale polylines of the same

190 target.

$191 \widehat{\operatorname{Sim}}_{L_{1}, L_{2}}^{\operatorname{Shp}}=\left(\int f\left(\theta_{L_{1}}-\theta_{L_{2}}\right) d s\right)^{\frac{1}{2}}=\left(\int_{0}^{1} T_{A}\left(L_{1}\right) d s-\int_{0}^{1} T_{A}\left(L_{2}\right) d s\right)^{\frac{1}{2}}$

192 where, $f\left(\theta_{L_{1}}-\theta_{L_{2}}\right)=\left\{\begin{array}{cc}\left|\theta_{L_{1}}-\theta_{L_{2}}\right| & \left(\left|\theta_{L_{1}}-\theta_{L_{2}}\right| \leq 180^{\circ}\right) \\ 360^{\circ}-\left|\theta_{L_{1}}-\theta_{L_{2}}\right| & \left(\left|\theta_{L_{1}}-\theta_{L_{2}}\right|>180^{\circ}\right)\end{array}\right.$.The $\widehat{\operatorname{Sim}}_{L_{1}, L_{2}}^{\operatorname{shp}}$ is

193 inversely correlated with the similarity degree between $L_{1}$ and $L_{2}$. If the angle is clockwise, $\alpha_{i}$ is

194 positive, and if the angle is counterclockwise, $\alpha_{i}^{\prime}$ is negative. The relation between $\alpha_{i}$ and $\alpha_{i}^{\prime}$ is that

$195 \alpha_{i}=360^{\circ}-\left|\alpha_{i}^{\prime}\right|$. For instance, if $\alpha_{i}^{\prime}=-18^{\circ}$, then $\alpha_{i}=360^{\circ}-18^{\circ}=342^{\circ}$.

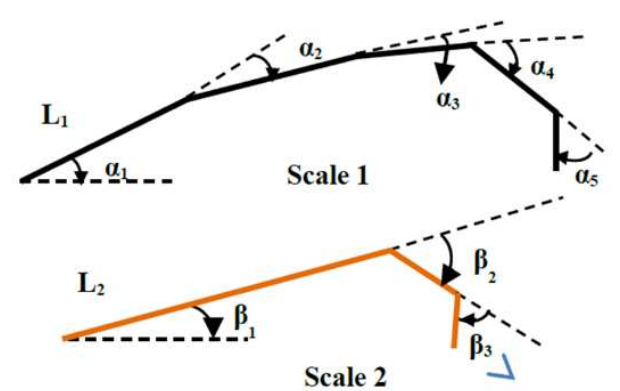

(a) Polyline $\mathrm{L}_{1}$ and its simplification result $\mathrm{L}_{2}$

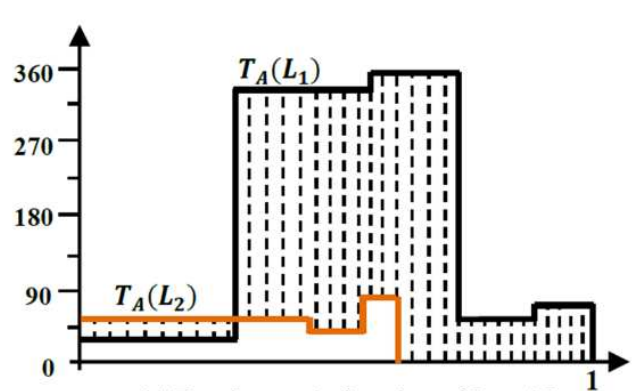

(b) Turning angle function of $\mathrm{L}_{1}$ and $\mathrm{L}_{2}$

Figure 3. Cumulative angle function of the multi-scale polylines $L_{1}$ and $L_{2}$. 

polyline entities. However, it is worth noting that the threshold of buffer radius is the

200 key to solving various research problems. According to the visual perception degree of 201 position deviation on the figure, buffer radius of the buffer-overlapped area is generally 202 set as 1 2 times of the minimum distance $(0.2 \mathrm{~mm})$ between two points on the target 203 scale map, e.g., if the target scale is $1: 250000$, then the buffer radius is $50 \mathrm{~m}$.

204 However, suppose we want to study the mechanism of spatial similarity varying with 205 map scale change. In that case, map scale change must be the unique independent 206 variable, and the same set of weights for different groups of datasets should be used to 207 eliminate the influence of weights variation, which may adversely influence the results. 208 Therefore, nine groups of weights of four stability factors are normalized to the same 209 group of weights (Table 1) by using the Sum-Product method.

210 Table 1. Normalized Weights of stability factors.

\begin{tabular}{ccccc}
\hline Impact factor & Distance & Sinuosity & Shape & Buffer-overlapped area \\
\hline Weight & 0.32 & 0.23 & 0.11 & 0.34 \\
\hline
\end{tabular}

211 The weights of sinuosity, distance, shape, buffer-overlapped area are $0.23,0.32,0.11$,

212 and 0.34 , the critical ranking of which is consistent with the weights obtained from the

213 opinions of experts based on spatial cognition experiment or the well-adopted criteria

214 (Chehreghan et al. 2016). The large weight value indicates that the corresponding factor 215 strongly influences the judgment of spatial similarity relations and vice versa. Thus, 
216 according to the value of the adopted weights, the buffer-overlapped area has the

217 greatest impact, followed by distance.

218 2.3. Construction of spatial similarity evaluation model

219 According to the definition and description of each index in Section 2.2, it is known to

220 all that some factors are positive indicators, such as distance, shape, sinuosity, while

221 others are negative, i.e., the larger $\widehat{\operatorname{sm}}_{L_{1}, L_{2}}^{D i s}$, indicates the less similarity between polylines,

222 while the $\widehat{\operatorname{sim}}_{L_{1}, L_{2}}^{B u f}$ is an opposite factor. Moreover, the units and dimensions of factors are

223 different, and their value varies greatly, which also has a great impact on the results.

224 Therefore, to eliminate the effects of negative factors and different units on results, the

225 similarity degree of these factors should be normalized to $[0,1]$ before determining the

226 spatial similarity evaluation model, which is achieved by using the most applicable

227 technique range standardization as follows:

228

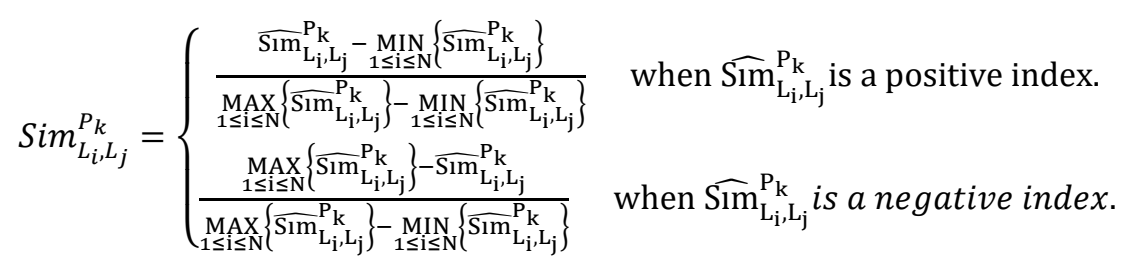

$229 \operatorname{Sim}_{\mathrm{L}_{\mathrm{i}}, \mathrm{L}_{\mathrm{j}}}^{\mathrm{P}_{\mathrm{k}}}$ is the similarity degree of the factor $\mathrm{P}_{\mathrm{k}}$ after normalized, where the large

$230 \operatorname{Sim}_{\mathrm{L}_{\mathrm{i}}, \mathrm{L}_{\mathrm{j}}}^{\mathrm{P}_{\mathrm{k}}}$ indicates the higher spatial similarity. $\mathrm{N}$ is the number of groups of datasets.

231 Finally, Equation (3) (Yan 2014) can be employed to calculate the spatial similarity

232 degree between polylines based on four factors.

$233 \operatorname{Sim}\left(\mathrm{L}_{\mathrm{i}}, \mathrm{L}_{\mathrm{j}}\right)=\sum_{k=1}^{n} \mathrm{~W}_{\mathrm{p}_{k}} \operatorname{Sim}_{\mathrm{L}_{\mathrm{i}}, \mathrm{L}_{\mathrm{j}}}^{\mathrm{p}_{\mathrm{j}}}$ 
where, $\operatorname{Sim}\left(L_{1}, L_{2}\right) \in(0,1], W_{p_{k}} \in[0,1], \mathrm{n}$ is the total number of impact factors, which is 4 .

\section{$235 \quad$ 2.4. Geometric accuracy assessment}

236 In the generalization of an individual polyline, the influence of simplification on the

237 geometric accuracy is mainly reflected in the change of geometric features and the

238 position of points on the polyline (Wu F et al. 2008, Chen et al. 2016). The variations

239 of the former are mainly represented by the changes in curve length and sinuosity. The

240 variations of the latter are mainly manifested as the global or local displacement before

241 and after the generalization of the polyline. Such variations are evaluated using the

242 standardized deviation of displacement (SMD), position error (PE), vector

243 displacement, area displacement, and buffer tolerance. Among these indices, the SMD

244 mainly evaluates the local maximum, and it is the most suitable index for comparing

245 the different simplification results of the same linear features (Wu et al. 2008).

246 Furthermore, PE is often used to evaluate the overall displacement (White 1985;

247 Mcmaster 1987). In this research work, the geometric accuracy of the simplified

248 polyline is evaluated based on the length ratio (LR) (Chehreghan and Abbaspour 2018),

249 sinuosity degree (SD) (Danlel and Yang 2014), SMD (Chen 2016), and PE.

250 By comparing the length ratio of the polyline, it can be seen how much the

251 simplification algorithm compresses the points on the polyline, and the ratio is inversely

252 correlated with the amount of compression ( $\mathrm{Wu} 2008)$. The degree of curvature can be

253 obtained by sum the included angles of adjacent straight lines on the polyline. If the 
254 length of polylines changes more and the sinuosity degree changes less, this indicates 255 that the geometric features of the simplified polyline are better maintained. The smaller 256 value of SMD represents a smaller displacement of a feature on the map. This implies a 257 lesser likeliness of intersection, which highlights that the spatial relationship is 258 maintained very well. Since the SMD can only be used to obtain the maximum value 259 of the polyline's local displacement, it cannot properly describe the overall 260 displacement. So, the PE is used to evaluate the positional accuracy of a polyline. The 261 smaller the PE value is, the better the accuracy of the point position on the curve is 262 maintained. Furthermore, the simplification result can effectively maintain the overall 263 shape characteristics of polylines.

\section{3. Experiments and Results}

265 According to different geographical features, the plain of China is divided into the

266 Northeast China Plain, the North China Plain, and the Yangtze Plain. Thirty-eight 267 groups of multi-scale datasets of rivers and roads, which include 1:10k, 1:25k, 1:50k,

$2681: 100 \mathrm{k}, 1: 250 \mathrm{k}$, and 1:500k, were selected to calculate the quantitative relationships 269 between $\mathrm{S}$ and $\mathrm{C}$. these data are divided into two major group categories: control and 270 experimental. The experimental group is composed of the multi-scale polylines datasets

271 from the same geographical feature area, each group of the dataset includes river and 272 road from the same sample area, and the former is composed of the multi-scale 273 polylines datasets from the different geographical feature areas, each group of the 
274 dataset includes river or road from the different sample areas. These multi-scale datasets 275 include 12 groups of river samples from the Huaibei Plain (Sample area I), which 276 belong to a part of the North China Plain, seven groups of river samples from the 277 Northeast China Plain (Sample area II). There are 19 groups of samples from the lower 278 Yangtze Plain (Sample area III), including 12 groups of the river and 7 groups of roads, 279 which are separately sampled from the Jianghuai Plain and the Yangtze River Delta 280 Plain, because both of which are part of the lower Yangtze Plain, we assume they share 281 the same geographic features. These multi-scale vector data, as mentioned above, were 282 derived from the data vectorization results of GF-2 images under different scales using 283 ArcGIS10.6. The GF-2 image is obtained by the fusion of Pan and multi-spectral image, 284 whose spatial resolution is $1 \mathrm{~m}$ and $4 \mathrm{~m}$, respectively. Multi-scale datasets of rivers and 285 roads, which include $1: 5 \mathrm{w}, 1: 10 \mathrm{w}, 1: 25 \mathrm{w}, 1: 50 \mathrm{w}$, and $1: 100 \mathrm{w}$, were derived from the 286 current results of the National Geomatics Center of China (NGCC). 


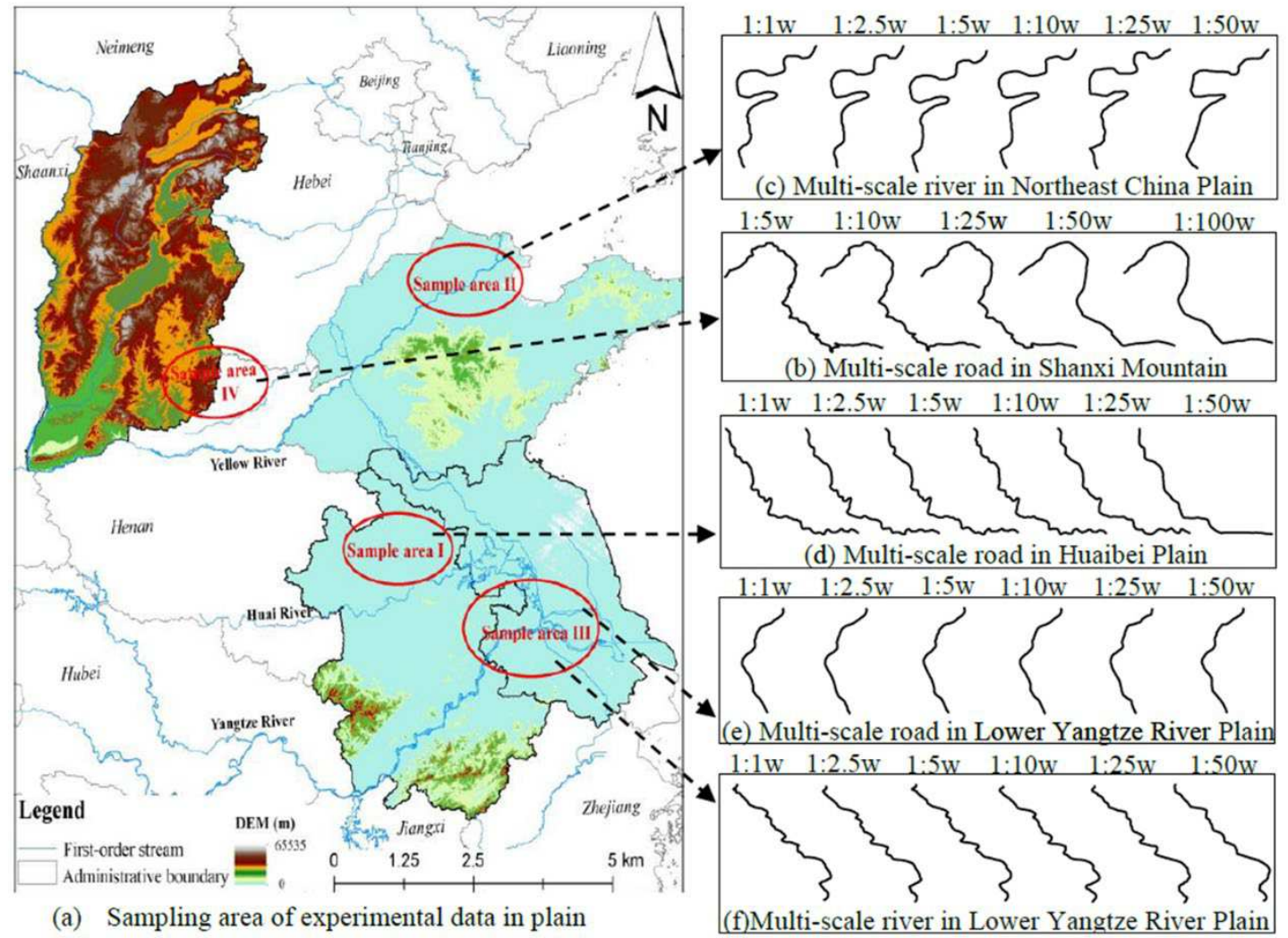

\subsection{Validation of the proposed spatial similarity evaluation}

\section{1 model}

292 It is qualitatively evident that the greater the map scale change, the smaller the spatial

293 similarity degree between multi-scale polylines. However, no specific quantitative

294 relationships between them are known by far, which hampers the complete automation

295 of the algorithm. There are five potential candidate functions to map this gap that can describe such changing trends, including linear function, polynomials, power functions, 
299 decreasing interval. Ultimately, only second-order polynomials should be considered.

300

$\left\{\begin{array}{l}\mathrm{y}=\mathrm{a}_{2} \mathrm{x}^{2}+\mathrm{a}_{1} \mathrm{x}+\mathrm{a}_{0} \quad\left(\mathrm{a}_{2}>0, \mathrm{x} \in\left[1,-\frac{a_{1}}{2 a_{2}}\right]\right) \\ y=a \operatorname{In}(x)+a_{0} \quad(\mathrm{a}<0, \mathrm{x} \geq 1) \\ y=a x^{b} \quad(\mathrm{a}>0,-1<\mathrm{b}<0, \mathrm{x} \geq 1) \\ y=a e^{b x} \quad(\mathrm{a}>0, \mathrm{~b}<0, \mathrm{x} \geq 1) \\ y=a x+b \quad(\mathrm{a}<0, \mathrm{x} \geq 1)\end{array}\right.$

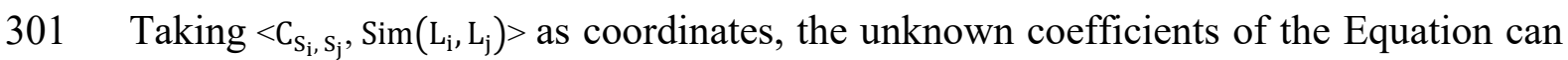

302 be determined using the curve fitting approach. Here, for $\operatorname{simplification}, \operatorname{Sim}\left(\mathrm{L}_{\mathrm{i}}, \mathrm{L}_{\mathrm{j}}\right)=$

$303 \mathrm{f}\left(\mathrm{C}_{\mathrm{S}_{\mathrm{i}}, \mathrm{S}_{\mathrm{j}}}\right)$ is replaced with $\mathrm{S}=\mathrm{f}(\mathrm{C})$. Statistical analysis is conducted based on Microsoft

304 Excel (V10.0) and the least square method $R^{2}\left(R^{2} \in[0,1]\right)$ is employed to choose the

305 best-fitted function (Yang et al. 2015). Considering that the five groups of datasets have

306 similar variation tendencies, multi-scale polylines from the Huaibei plain are taken as

307 an example (Figure 5).
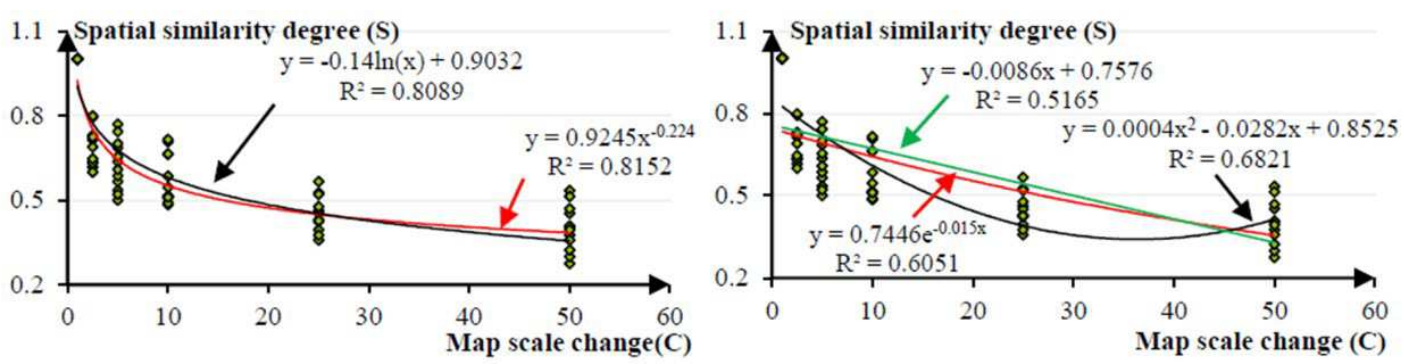

309 Figure 5. Fitting results of five functions and its $\mathbf{R}^{2}$ of multi-scale polylines from the Huaibei plain.

$310 \mathrm{R}^{2}$, as a good indicator, often is used to compare the candidate functions. The larger

311 value of $\mathrm{R}^{2}$ always indicates the better fitting curve. As can be clearly seen from Figure

3125 , the $R^{2}$ of the power function is closest to $1\left(R^{2}=0.8152\right)$, which achieves the best 
313 fitting relationship of the curve among all candidates. Therefore, Equation (5) is chosen

314 as a quantitative model for describing the relationships between spatial similarity

315 degree and map scale change of individual polylines. This conclusion is consistent with

316 Yan's conclusion (Yan 2014).

$317 \operatorname{Sim}\left(\mathrm{L}_{\mathrm{i}}, \mathrm{L}_{\mathrm{j}}\right)=\left\{\begin{array}{l}1, \text { if } \mathrm{L}_{1} \text { and } \mathrm{L}_{2} \text { are straight line, otherwise } \\ \mathrm{aC}_{\left(\mathrm{L}_{\mathrm{i}}, \mathrm{L}_{\mathrm{j}}\right)} \mathrm{b}\end{array}\right.$

318 where, $a>0,-1<b<0 ; \operatorname{Sim}\left(\mathrm{L}_{\mathrm{i}}, \mathrm{L}_{\mathrm{j}}\right) \in(0,1], \mathrm{C}_{\left(\mathrm{L}_{\mathrm{i}}, \mathrm{L}_{\mathrm{j}}\right)} \in[1,+\infty)$.

319 To validate the reliability of the proposed model, this paper selected five groups of

320 vector sampling multi-scale polylines from previous research of Yan (2015) and

321 Chehreghan (2016). Figure 6 shows the fitting results of the proposed model, and Table

3222 shows the accuracy comparison result with the existing evaluation model (Yan 2014).
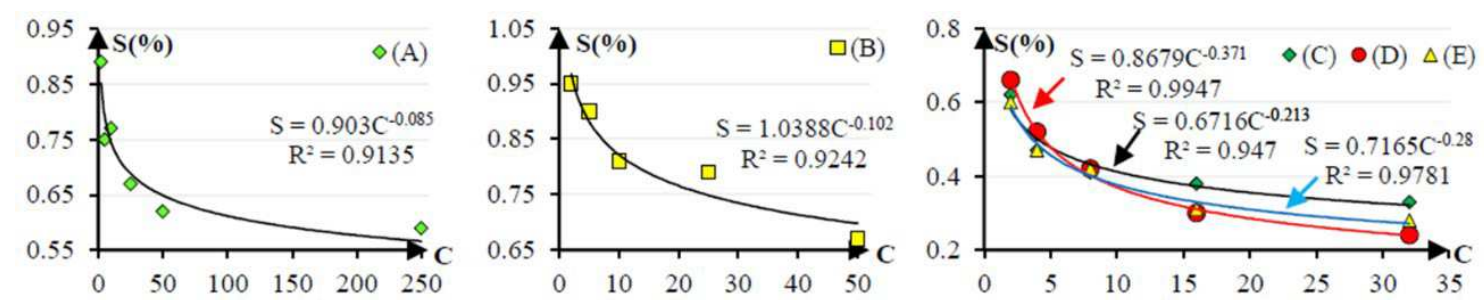

where, (A), (C), and (E) corresponds to Figure 2, Figure 3, and Figure 4 of Yan's research (2015), respectively; (B) and (D) correspond to Figure11 and Figure 13 of Chehreghan's research (2016), respectively.

324 Figure 6. Quantitative relationships fitting results of validation data between S and C.

325 Table 2. Accuracy comparison result with the existing evaluation model.

\begin{tabular}{cccc}
\hline Group & Previous evaluation & Proposed evaluation & Accuracy improvement \\
\hline (A) & $\mathrm{S}=1.0164 \mathrm{C}^{-0.3427}$, & $\mathrm{S}=0.903 \mathrm{C}^{-0.085}, \mathrm{R}^{2}=0.9135$ & $4.16 \%$ \\
(C) & & $\mathrm{S}=0.6716 \mathrm{C}^{-0.213}, \mathrm{R}^{2}=0.9470$ & $7.98 \%$ \\
(E) & $\mathrm{R}^{2}=0.8770$ & $\mathrm{~S}=0.7165 \mathrm{C}^{-0.28}, \mathrm{R}^{2}=0.9781$ & $11.52 \%$ \\
\hline
\end{tabular}


326 Compared with the previous model, the fitting accuracy of the evaluation model 327 proposed in this paper is improved by $4.16 \% \sim 11.52 \%$. However, compared with 328 Chehreghan's conclusion, there is a nonlinear power function relationship between 329 spatial similarity and map scale change (Figure 6(B), (D)). Although the trend of three 330 groups of the multi-scale polyline (Figure $6(\mathrm{~A}),(\mathrm{C}),(\mathrm{E}))$ is the same, they cannot be

331 described using the same power function whether they are the same ground objects of 332 different types or the different ground objects of same type, which is some different 333 with Yan's and Chehreghan's conclusion.

334 In order to further verify whether these quantitative relationships can be fitted using the 335 same function with the same coefficients, this paper selects 38 groups of the dataset of 336 different plains, and the curve fitting results are shown in Figure 7. 

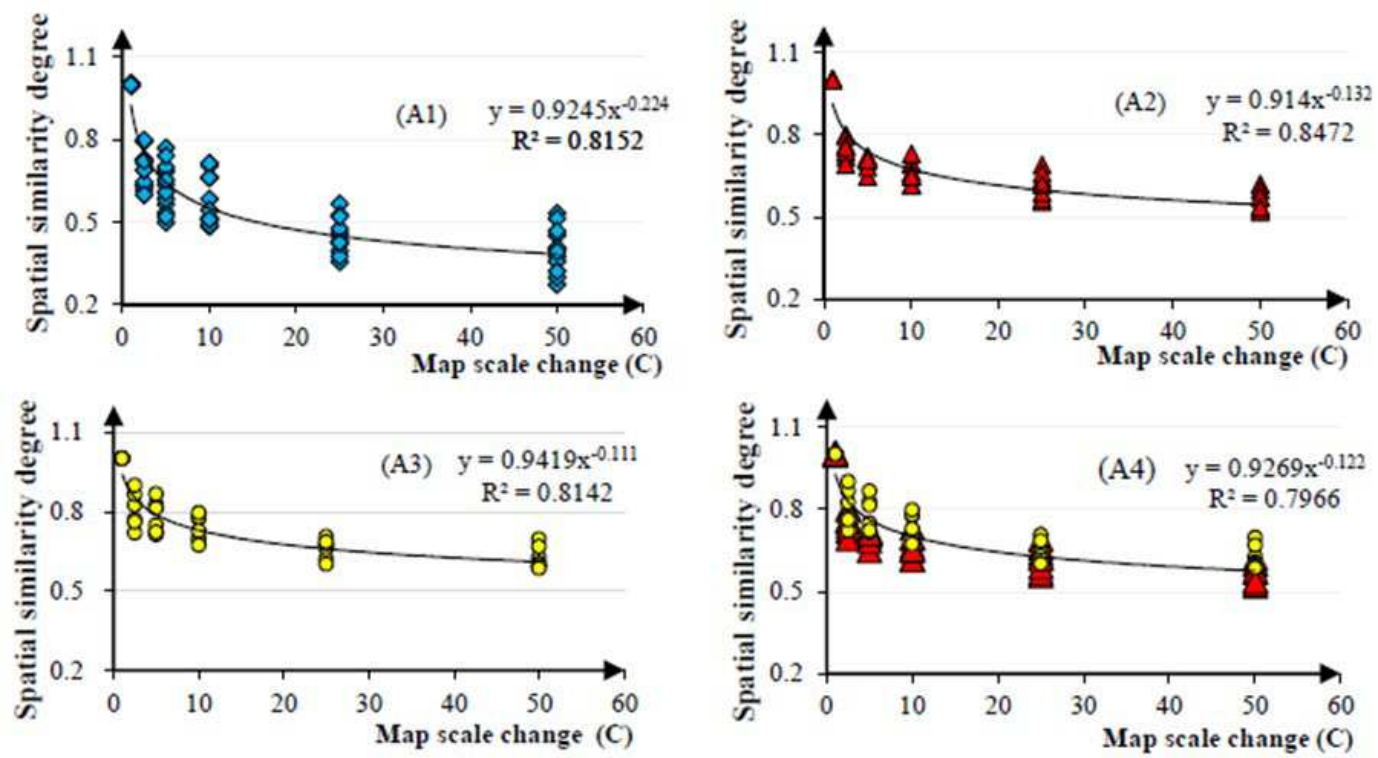

(A) Experimental group
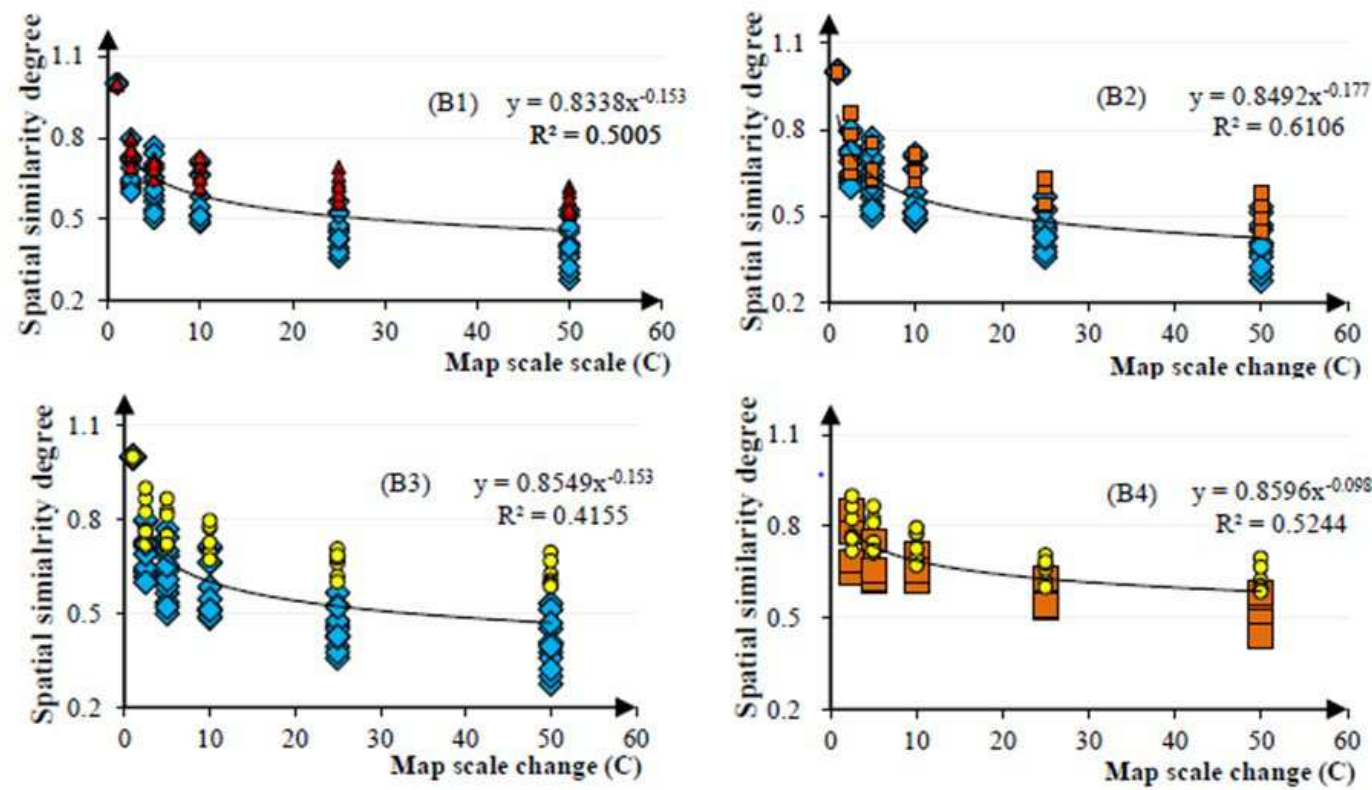

(B) Control group

$\diamond$ Huaibei Plain(River) O Lower Yangtze Plain(Road) $\quad \boldsymbol{\Delta}$ Lower Yangtze Plain(River) $\quad$ Northeast China Plain (River)

Figure 7. Fitting results between $\mathrm{S}$ and $\mathrm{C}$ of the multi-scale polylines.

339 Figure $7(\mathrm{~A})$ shows the fitting results between $\mathrm{S}$ and $\mathrm{C}$ of multi-scale individual

340 polylines from the same geographical feature area, and Figure 7(B) shows the fitting

341 result of multi-scale individual polylines from the different geographical feature areas. 
342 Through the comparative analysis of the above fitting results in Figure 7, it can be seen

343 clearly that the relationship between spatial similarity degree and map scale change of

344 different groups of the dataset from the same geographical feature plain can be

345 described using the same power function curve with the same parameters, e.g., in Figure

346 7(A1), 12 groups of multi-scale polylines of the river are all from the Huaibei Plain, the

347 quantitative relationship between $\mathrm{S}$ and $\mathrm{C}$ can be fitted using the same power function

$348 \mathrm{~S}=0.9245 \mathrm{C}^{-0.224}$, and the fitting accuracy $\mathrm{R}^{2}$ is up to 0.8152 . Moreover, 19 groups of

349 multi-scale polylines of river and road are all from the Lower Yangtze Plain in Figure

3507 (A4), although they are the different types of the polyline, and some groups of

351 polylines of which are sampled from the Jianghuai Plain, while others are sampled from

352 the Yangtze River Delta Plain, the fitting accuracy is also up to 0.7966. However,

353 Figure 7(B) shows that no matter whether the polylines are the same or different type(s)

354 of features, the coefficients of their power function are entirely different for polylines

355 from the different geographical feature areas. For example, as shown in Figure 7(B1),

35612 groups of multi-scale polylines are from the Huaibei Plain, and the other 12 groups

357 of multi-scale polylines are from the Lower Yangtze Plain, both of them are the same

358 type of ground objects, but the fitting accuracy of power function is only 0.5005 . In

359 Figure 7(B3), 12 groups of multi-scale polyline of the river are from the Huaibei Plain,

360 and seven groups of multi-scale polyline of the road are from the Lower Yangtze Plain;

361 both are the different types of ground objects, the fitting accuracy is also only 0.4155 . 


\subsection{Determination of distance threshold}

363 The essence of the DP algorithm is to compare the straight-line distance connecting the

364 start point and endpoint with the optimal distance threshold. Therefore, this part aims

365 to find the optimal distance threshold, which determines the degree of the polyline to

366 be simplified, corresponding to map scale change to realize the completely automatic

367 generalization of polylines.

368 It is qualitatively evident that the greater the map scale change, the greater the optimal

369 distance threshold of DP. Hence, three potential candidate functions can be employed

370 to fit the changing trend between $\lambda$ and $\mathrm{C}$ : polynomial, linear Equation, and logarithmic

371 function. Since the other polynomials have n-1 inflection point(s), e.g., cubic

372 polynomial, which indicates that the curve is not monotonic. Hence, only the second-

373 order polynomial $(\mathrm{x} \in[0,-b / 2 a])$ satisfies the variation tendency that the dependent

374 variable increases with the independent variable. Ultimately, only quadratic polynomial,

375 linear Equation, and logarithmic function are considered (Equation (6)).

$$
\left.\begin{array}{ll}
y=a x^{2}+b x+d & \left(x \in\left[1,-\frac{b}{2 a}\right]\right) \\
y=a \operatorname{In}(x)+b & (x \geq 1) \\
y=a x+b & (x \geq 1)
\end{array}\right\}
$$

377 According to the principle described in section 2.1, the point pairs $(\mathrm{C}, \mathrm{T})$ of the optimal

378 distance threshold and map scale change are continuously recorded with the gradual

379 generalization of the polyline. The fitting results between them are shown in Figure 8. 


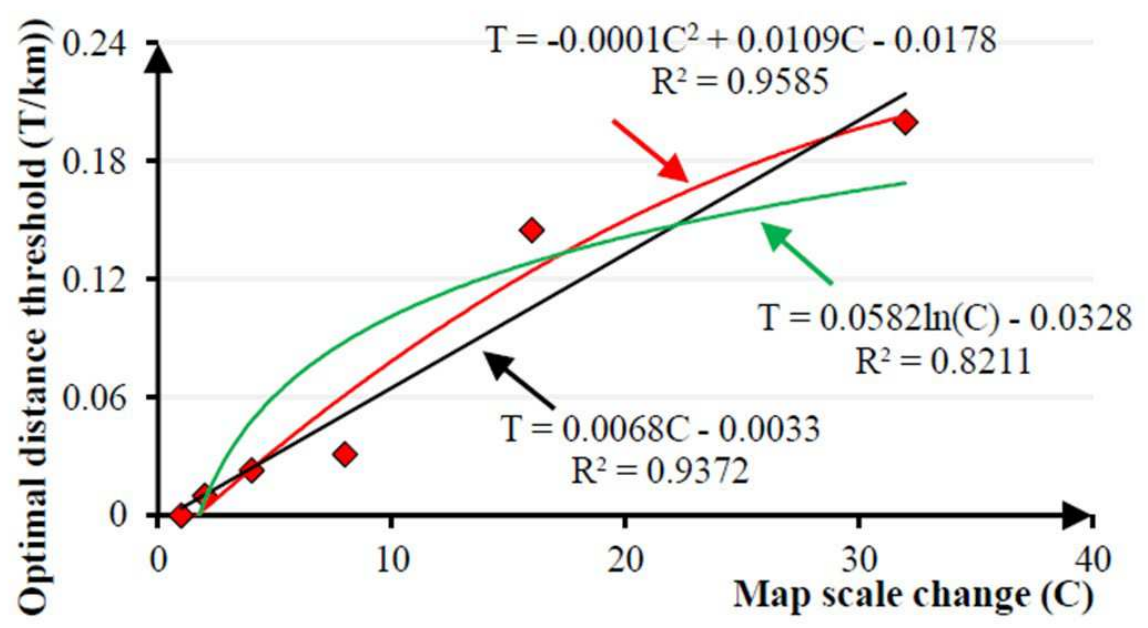

381 Figure 8. Optimization result of candidate functions between $\mathbf{T}$ and $\mathbf{C}$.

382 As can be clearly seen from Figure 8 , the $\mathrm{R}^{2}$ of the unary quadratic function is closest

383 to $1\left(\mathrm{R}^{2}=0.9585\right)$, which achieves the best fitting relationship of the curve among all the

384 candidates. Therefore, the unary quadratic function (Equation (7)) is chosen as the

385 quantitative model for describing the relationships between $\mathrm{T}$ and $\mathrm{C}$.

$386 \quad\left\{\begin{array}{l}\mathrm{T}=0 \quad(C=1) \\ \mathrm{T}=\mathrm{a} C^{2}+b C+d\left(\mathrm{a}<0, \mathrm{C} \in\left(1, \max \left\{\mathrm{C}_{\mathrm{i}}\right\}\right]\right)\end{array}\right.$

387 In order to further verify whether the quantitative relationships between $\mathrm{T}$ and $\mathrm{C}$ can be

388 fitted using the same function with the same coefficients, the experimental datasets of

389 this part are divided into two parts: control and experimental. The former consists of

390 multi-scale datasets from the different geographical feature areas, and the quadratic

391 functions are shown in Figure 9(a). The latter consists of multi-scale datasets from the

392 different geographical feature areas, and the quadratic functions are shown in Figure 393 9(b). 

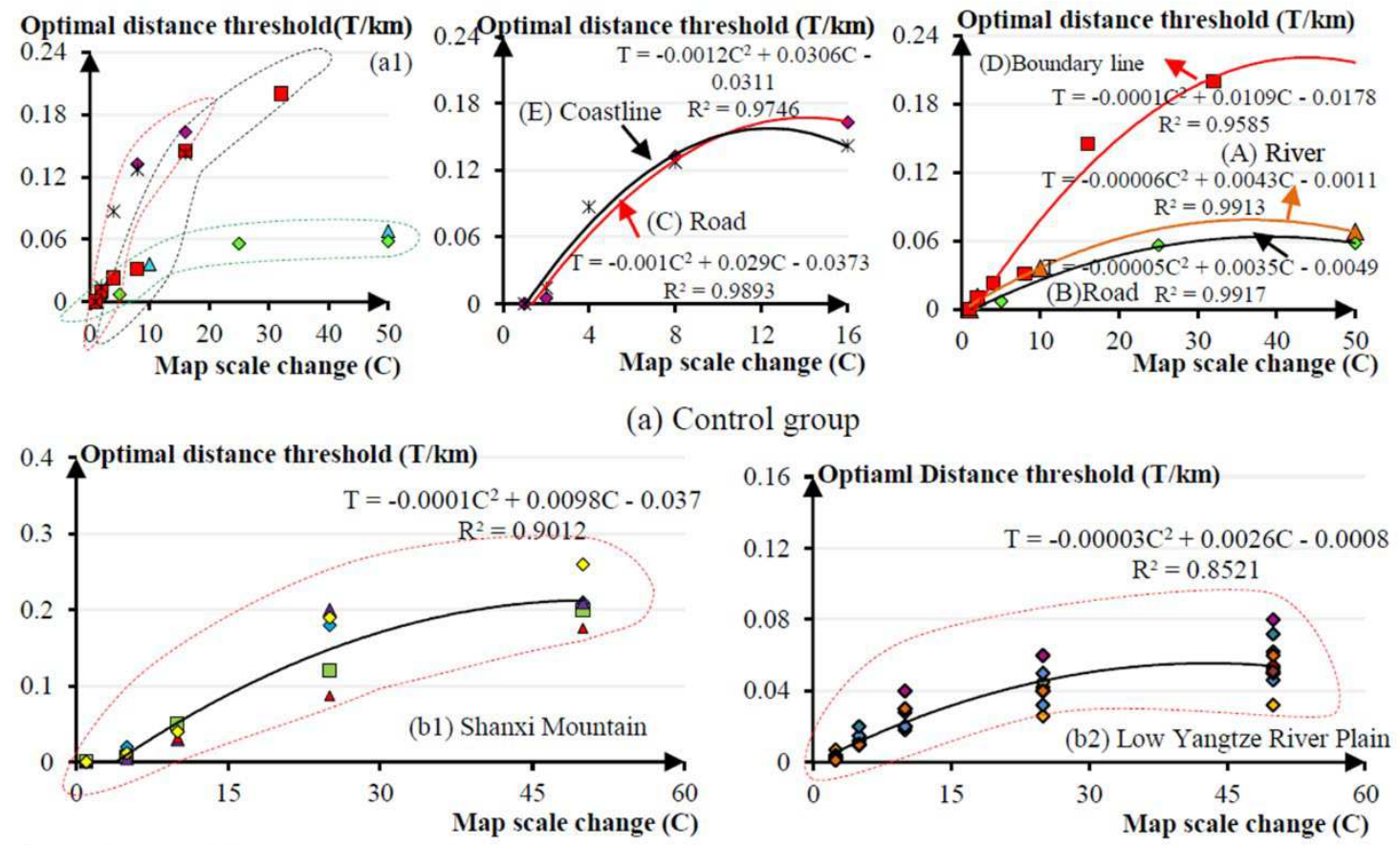

$\diamond$ Road $\triangle$ River $\quad \square$ boundary

(b) Experimental group

Figure 9. Quantitative relationship fitting results between $\mathrm{T}$ and $\mathrm{C}$ of multi-scale polylines.

396 It can be clearly seen from Figure 9(a) that the $\mathrm{R}^{2}$ of each dataset in the control group

397 is no less than 0.9585 , and the maximum is up to 0.9917 , indicating a very high fitting

398 accuracy. However, Figure 9(a1) shows that no matter whether the polylines are the

399 same or different type(s) of features (e.g., B and C both are roads; D and E are the

400 different types of features of the real world), the coefficients of their unary quadratic

401 function are entirely different for polylines from the different geographical feature area.

402 Consequently, these results convincingly demonstrate that it is unreasonable to describe

403 all five groups of datasets using the same single quadratic Equation with the same

404 coefficients; besides, $\mathrm{R}^{2}$ is only 0.4636 . Therefore, it is impossible to simultaneously

405 realize the completely automatic generalization of all polylines using the same optimal

406 distance threshold. 
407 Based on the results presented in Figure 9(b), the first group of experimental data which 408 is consisted of four groups of multi-scale polyline from Shanxi Mountain (Figure 9(b1)), 409 the other consists of 19 groups of multi-scale polylines from the Lower Yangtze River 410 Plain (Figure 9(b2)). The $\mathrm{R}^{2}$ values of the two groups of datasets are not less than 0.8521 , 411 with the best in the first groups $\left(\mathrm{R}^{2}=0.9012\right)$. Hence, the fitting accuracy is satisfactory. 412 i.e., it is affirmative to realize the completely automatic generalization of all two groups 413 of sampling datasets, respectively. Therefore, these results convincingly demonstrate 414 that it is reasonable to realize the complete automation of the DP generalization 415 algorithm for the polylines from the same geographical feature area. Therefore, 416 polylines from the same geographical feature area are the objects of the following 417 research.

\section{3.3. Automated generalization of a polyline}

419 Taking the multi-scale polylines from the Shanxi Mountain as an example, the 420 theoretical optimal distance threshold is determined if the original and target map scales 421 are given (Table 3). For example, suppose the original and target map scales are $4221: 50000,1: 250000$, respectively, and then the map scale change $C$ is 5 . Hence, the 423 optimal distance threshold $\mathrm{T}$ will be $0.0095 \mathrm{~km}$. Afterward, six groups of experimental 424 datasets are automatically generalized using DP based on the corresponding theoretical 425 optimal distance threshold. 


\begin{tabular}{ccccccccccccc}
\hline \multicolumn{4}{c}{$\mathrm{T}=-0.0001 \mathrm{C}^{2}+0.0098 \mathrm{C}-0.037$} & $(\mathrm{C} \in(1,50])$ & \multicolumn{1}{c}{$\mathrm{T}=-0.00003 \mathrm{C}^{2}+0.0026 \mathrm{C}-0.0008(\mathrm{C} \in(1,50])$} \\
\hline 1 & $\mathrm{C}$ & 5 & 10 & 25 & 50 & 2 & $\mathrm{C}$ & 2.5 & 5 & 10 & 25 & 50 \\
& $\mathrm{~T} / \mathrm{km}$ & 0.0095 & 0.0510 & 0.1455 & 0.2030 & & $\mathrm{~T} / \mathrm{km}$ & 0.0056 & 0.0114 & 0.0222 & 0.0455 & 0.0542 \\
\hline
\end{tabular}

427 Figure 10 shows the results of the experimental datasets based on the proposed method.
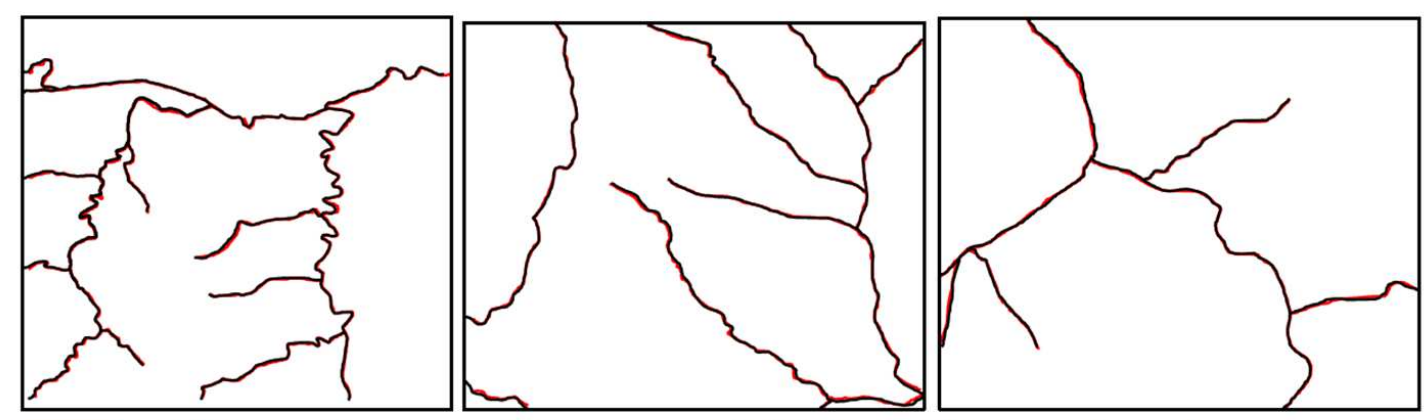

1:250000 (Simplification result $(\mathrm{L}))$

1:250000 (Standard target scale map $\left(\mathrm{L}_{\mathrm{M} 2}\right)$ )

(a) Comparison graph of river automatic generalization result.

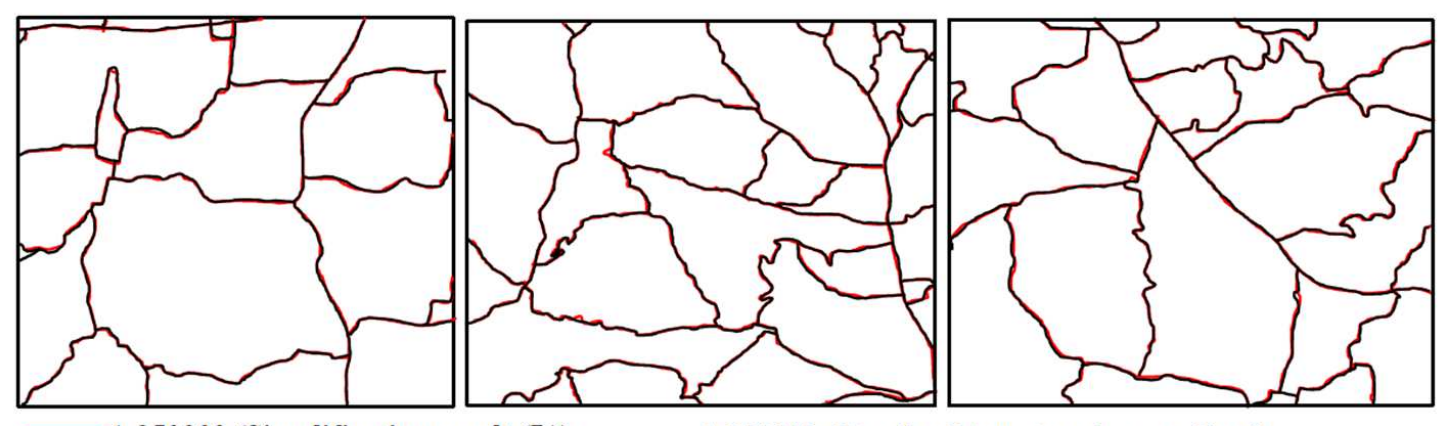

1:250000 (Simplification result $(\mathrm{L})) \quad$ 1:250000 (Standard target scale map (LM2))

(b) Comparison graph of road automatic generalization result.

429 Figure 10. Experiment results of the different datasets from the same geographical feature area.

\subsection{Accuracy evaluation}

431 The proposed model is validated for its acceptability and consistency. In this regard,

432 length ratio (LR), Sinuosity ratio (SR), PE, and SMD are employed to evaluate the

433 geometric and position accuracy of generalization results (Wu et al. 2008).

434 Table 4. Geometric accuracy comparison between simplification results and available data.

\begin{tabular}{|c|c|c|c|c|c|c|c|c|}
\hline \multirow{2}{*}{ Features } & \multicolumn{4}{|c|}{ Target scale reference Data $\left(\mathrm{L}_{\mathrm{M} 2}\right)$} & \multicolumn{4}{|c|}{ Simplification results of proposed method (L) } \\
\hline & LR & SR & PE\km & SMD (\%) & $\mathrm{LR}^{\prime}$ & $\mathrm{SR}^{\prime}$ & $\mathrm{PE}^{\prime} \mathrm{km}$ & SMD' $^{\prime}$ \\
\hline Road1 & 0.9901 & 0.9145 & 0.0211 & 0.4661 & 0.9915 & 0.9004 & 0.0578 & 0.4565 \\
\hline
\end{tabular}




\begin{tabular}{lllllllll}
\hline Road2 & 0.9540 & 0.9227 & 0.0321 & 0.5334 & 0.9668 & 0.9154 & 0.0772 & 0.5102 \\
Road3 & 0.9759 & 0.9032 & 0.0415 & 0.6029 & 0.9869 & 0.9056 & 0.0295 & 0.5621 \\
River1 & 0.9983 & 0.7708 & 0.0478 & 0.6046 & 0.9983 & 0.7658 & 0.0448 & 0.5803 \\
River2 & 0.9832 & 0.8827 & 0.0021 & 0.5662 & 0.9874 & 0.8789 & 0.0398 & 0.5663 \\
River3 & 0.9966 & 0.8678 & 0.0101 & 0.6211 & 0.9975 & 0.8339 & 0.0641 & 0.6128 \\
\hline
\end{tabular}

435 Table 4 also reveals that comparing with $\mathrm{L}_{\mathrm{M} 2}, \triangle \mathrm{LR}$ of samples is less than zero, and

$436 \triangle \mathrm{SR}$ is almost greater than zero, the error of the compression amount $(\triangle \mathrm{LR})$, but the

437 sinuosity degree change rate $(\triangle \mathrm{SR})$ of the road and river are the smallest. It highlights

438 that the compression amount (LR) and the sinuosity degree change rates (SR) of L are

439 consistent with $\mathrm{L}_{\mathrm{M} 2}$, and the proposed approaches can better maintain the geometric

440 features of road and river; the data compression effect is superior.

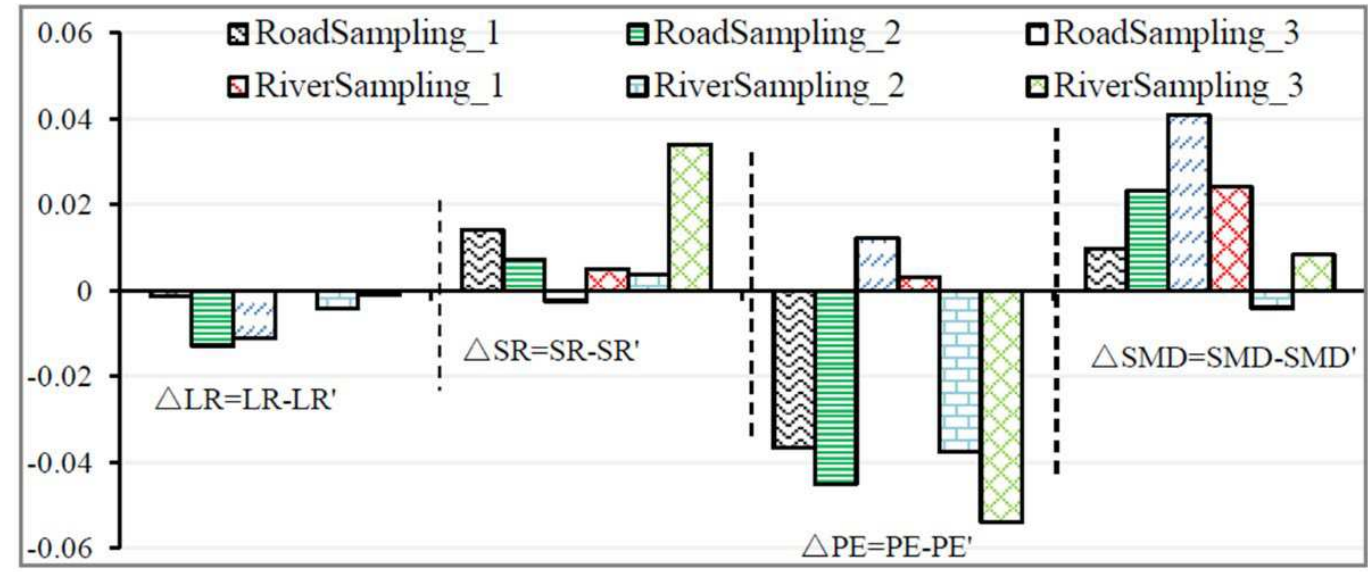

442 Figure 11. Error rate comparison of geometric and position accuracy of simplification results

443 The road and river simplification results are comparable to the standard target scale

444 polyline in terms of maintaining the position accuracy (Figure 11). Compared with the

445 standard target scale polylines, the position errors of road and river are significantly

446 superior to the error-index values of LM2 except for two groups of samplings, but the

447 maximum position errors are only 0.012. It highlights that the simplification results of

448 the proposed method could better maintain the global position accuracy of polylines. 
449 As shown in Table 4, the SMD is almost greater than zero, but the maximum of SMD 450 of L only is up to 0.0408 , i.e., the index SMD also indicates that the simplification

451 results of the proposed method could better maintain the local position accuracy of 452 polylines. In summary, the proposed method effectively maintains the local and global 453 shape characteristics of roads and rivers.

\section{4. Discussions}

455 Compared with special subjective parameter determination algorithms (Douglas and 456 Peucker 1973; Li and Openshaw 1992; Ai et al. 2016; Qian 2017), which require 457 humans' constant interference, this paper constructed the quantitative relationships 458 between the optimal distance threshold and map scale change using the spatial 459 similarity relations of polylines based on two groups of control experiments, which 460 consider polylines from the same or different geographical feature areas. Finally, based 461 on the theoretical optimal distance, the polylines from the same geographical feature 462 areas are completely automatically generalized using the Douglas-Peucker algorithm.

463 First, compared with the linear Equation and logarithmic function, the $\mathrm{R}^{2}$ of the unary 464 quadratic function is no less than 0.8521 . Therefore, the unary quadratic functions are 465 the best as the quantitative model for describing the relationships between the optimal 466 distance threshold and map scale change.

467 Second, no matter whether the polylines are from the same or different geographical 468 feature area(s), the unary quadratic function can be employed to describe the 
quantitative relationship between the optimal distance threshold and map scale change.

470 However, coefficients $a, b$, and $c$ are the same for the polylines from the same

471 geographical feature area, whereas the coefficients are different for the polylines from

472 the different geographical feature areas. Similar results were also found between the 473 similarity degree and map scale change. Thus, there are two main objective factors 474 affecting map generalization: scale and regional geographic characteristics. This 475 conclusion illustrates those regional geographic characteristics influence the 476 determination of the quantitative relationship between the optimal distance threshold 477 and map scale change. Therefore, it is reasonable to generalize the polylines from the 478 same geographical area using the same single optimal distance threshold.

479 Third, according to the quantitative functional relation of the polylines from the same 480 geographical feature area, if the map scale change is given, the theoretical optimal 481 distance threshold can be calculated, and vice versa. Compared with semi-automatic or 482 non-automatic polyline simplification algorithm (Gu 2016; Qian HZ 2017; Li CM 483 2017), this not only can facilitate the automation of map generalization software but 484 avoid human intervention in the process of map generalization, which will promote the 485 efficiency of the algorithm and the accuracy of map generalized results.

486 Last, this paper validated the acceptability and consistency of the geometric and 487 position accuracy by comparing them with the standard target scale polyline. The 488 experiment results indicate that the SMD of the road and the river simplified by the 489 proposed method is better than the s standard target scale polyline, i.e., geometric 
490 features retention of the proposed method is more superior. This conclusion is

491 consistent with the conclusions drawn by $\mathrm{Wu}$ (2008), who compared the Douglas-

492 Peucker, Li-Openshaw, Circle, and asymptotic algorithms. Therefore, it can be

493 concluded that the proposed method can better maintain the geometric features and

494 shape characteristics of polylines on the whole in terms of geometric and position

495 accuracy. In this paper, multi-scale individual polylines are taken as research objects.

496 However, the generalization of polyline group objects is not only dependent on the

497 features of ground features; it is closely related to the surrounding ground features.

498 Therefore, due to the defects of the DP algorithm itself, the simplification results may

499 intersect or intersect with other adjacent features that are close in distance, e.g., two

500 adjacent contours with a smaller distance between them simplified by the DP algorithm

501 may intersect. It is recommended that future work should conduct extensive

502 experiments over polyline group objects, e.g., contour cluster, road network, reticulate

503 drainage.

\section{5. Conclusions}

505 This paper proposed a model, which describes the quantitative relationships between

506 the optimal distance threshold and map scale change, to minimize the uncertainties in

507 the complete automation of the Douglas-Peucker algorithm. It can calculate the

508 theoretical optimal distance threshold of the polylines from the same geographical

509 feature area taking map scale change as the only independent variable and vice versa. 
510 It is indicated that realizing the complete automation of the Douglas-Peucker algorithm

511 is affirmative for the polylines from the same geographical feature area. The findings

512 of the current study not merely provide an idea and method for realizing the complete

513 automation of map generalization by looking for the quantitative relationship between

514 the parameter of algorithm and map scale change but also facilitate the automation of

515 map generalization algorithms and system of polyline and improve the accuracy of map

516 generalized results. The proposed model optimizes algorithm threshold settings,

517 eventually reducing the inevitable differences between automated-simplified and 518 cartographers' ratified simplification results. Reduced uncertainties in realizing the

519 Douglas-Peucker Algorithm automation would support improved spatial data matching 520 and establish a vector map database.

\section{$521 \quad$ References}

522 Ai, T.H., Shu, K., Yang, M., et al., 2016. Envelope generation and simplification of 523 polylines using Delaunay triangulation. International Journal of Geographical $524 \quad$ Information Science, 31(2), 297-319.

525 Chen, J. N., Qian, H. Z., Wang, X., et al. 2016. Geometric accuracy assessment of linear 526 features' simplification algorithm. Geomatics and Information Science of Wuhan 527 University, 45(4):486-493. [in Chinese]

528 Chehreghan, A., Abbaspour, R.A., 2018. A geometric-based approach for road 529 matching on multi-scale datasets using a genetic algorithm. Cartography and $530 \quad$ Geographic Information Science, 45(3), 155-269. 
531 Chehreghan, A., Abbaspour, R.A., 2016. An assessment of spatial similarity degree 532 between polylines on multi-scale, multi-source maps. Geocarto International, $533 \quad 32(5), 471-487$.

534 Danlel, P.A., Yang, P., 2014. Quantitative methods for comparing different polyline 535 models. Journal of Geographic Information System, 6, 88-98.

536 Deng, M., Li, Z.L., Chen, X.Y., 2007. Extended Hausdorff distance for spatial objects 537 in GIS. International Journal of Geographical Information Science, 21(4):459-475.

538 Douglas, D.H, Peucker, T. K., 1973. Algorithms for the reduction of the number of 539 points required to represent a digitized line or its caricature. The Canadian 540 Cartographer, 2(10):112-122.

541 Efrat, A. et al. Geometry helps in bottleneck matching and related problems. $542 \quad$ Algorithmica, 2001, 31 (1):1-28.

543 Gu, T., Chen, X. Y., Liu, C.Q., 2016. A modified line simplification method combined 544 Douglas-Peucker with Li-Openshaw. Journal of East China University of 545 Technology, 39(4):396-400. [in Chinese]

546 Hershberger, J., Snoeyink, J., 1992. Speeding up the Douglas-Peucker line-simplication 547 algorithm. In: Proceedings of the fifth symposium on data handling UBC 548 Technical report TR-92-07, pp:134-143.

549 Hughes, G.F., 1968. On the mean accuracy of statistical pattern recognizers. IEEE Trans. $550 \quad$ Inf. Theory, 14, 55-63.

551 Kronenfeld, B. J., Stanislawsuki, L.V., Buttenfield, B.P., et al, .2020. Simplification of 
polylines by segment collapse:minimizing areal displacement while preserving area. International Journal of Cartography, 6(1):22-46.

Li, Z. L., Openshaw, S., 1992. Algorithms for automated line generalization based on a natural principle of objective generalization. International Journal of Geographic Information Systems, 16(5):373-389.

Li, C. M., Guo, P.P., Yin, Y., et al., 2017. A line simplification algorithm considering spatial relations between two lines. Acta Geodaetica et Cartographica Sinica,

560 Li, Z. L., Sui, H.G., 2000. An integrated technique for automated generalization of contour maps. The Cartographic Journal. 37(1):29-37.

Li, ZX, Zhai, J.S., Wu, F., 2018. Shape similarity assessment method for coastline generalization. ISPRS International Journal of GeoInformation, 7(7):283.

564 Li, B., Fonseca, F.T., 2006. TDD: A comprehensive model for qualitative spatial similarity assessment. Spatial Cognition and Computation, 6(1):31-61.

566 Makris, A., Silva, C.L.d., Bogorny, V. et al. Evaluating the effect of compressing algorithms for trajectory similarity and classification problems. Geoinformatica 25, 679-711 (2021). https://doi.org/10.1007/s10707-021-00434-1.

Mcmaster, R. B., 1986. A statistical analysis of mathematical measures for linear simplification. The American Cartographer, 13(2):103-116.

571 Mcmaster, R. B., 1987. Automated line generalization. Cartographica, 24(2):74-111.

572 Nayyeri, A. et al., Computing the Fréchet Distance between polygons with holes. In M. 

Languages, and Programming: 42nd International Colloquium, ICALP 2015, 6Heidelberg, 997-1009.

Olteanu-Raimond, A., Mustière, S., Ruas, A., 2015. Knowledge formalisation for vector data matching using belief theory. Journal of Spatial Information Science, 10:2146.

Olteanu-Raimond, A., et al., 2008. Data matching - a matter of belief. In: Ruas A, Gold C, editors. Headway in spatial data handling. Lecture notes in geoinformation and cartography. Berlin: Springer, p. 501-519.

Peuquet, D.J., 1992. An algorithm for calculating minimum euclidean distance between two geographic features. Computers \& Geosciences, 18 (8), 989-1001.

Qian, H. Z., He, H. W., Wang, X., et al. Line feature simiplification method based on Bend group division[J]. Geomatics and Information Science of Wuhan University, 2017, 42(8):1096-1103. [in Chinese]

Ramer, U., 1972. An iterative procedure for the polygonal approximation of plane curves. Computer Graph Image Process, 1(3):244-256.

Saalfeld, A. (1999). Topologically consistent line simplification with the DouglasPeucker algorithm. Cartography and Geographic Information Science, 26(1), 7-18.

Sama,1 A., Seth, S., Cueto, K., 2004. A feature-based approach to conflation of geospatial sources. International Journal of Geographical Information Science, 
595 Sandu Popa, I., Zeitouni, K., Oria, V. et al. Spatio-temporal compression of trajectories in road networks. Geoinformatica 19, 117-145 $(2015)$ https://doi.org/10.1007/s10707-014-0208-4.

598 Tong, X.H., Liang, D., Jin, Y.M., 2014. A linear road object matching method for 599 conflation based on optimization and logistic regression. International Journal of 600 Geographical Information Science, 28(4):824-846.

601 Wu, F., Zhu, K. P.. 2008. Geometric accuracy assessment of linear features' simplification algorithm. Geomatics and Information Science of Wuhan University,

603 33(6):600-603. [in Chinese]

604 White, E. R., 1985. Assessment of line-generalization algorithms using characteristic 605 points. The American Cartographer, 12(1):17-28.

606 Yan, H. W., 2010. Fundamental Theories of Spatial Similarity Relations in Multi-scale 607 Map Spaces. Chinese Geographical Science, 20(1):18-22.

608 Yan, H. W., Li, J., 2014. Spatial similarity relations in multi-scale map spaces [M]. 609 Springer International Publishing Switzerland.

610 Yan, H. W., Shen, Y. Z., Li, J., 2016. Approach to calculating spatial similarity degrees 611 of the same river basin networks on multi-scale maps. Geocarto International, $612 \quad 31(7): 765-782$.

613 Yan, H. W., 2015. Quantitative relations between spatial similarity degree and map scale 614 change of individual linear objects in multi-scale map spaces. Geocarto 
616 Yang, W. F., Yan, H. W., Jonathan, L., 2015. Formula for calculating spatial similarity 617 degrees between poing clouds on multi-scale maps taking map scale change as the 618 only independent variable. Geodesy and Geodynamics, 2(6):113-125.

619 Shen, Y. L., Ai, T. H., Wang, L., et al. 2018. A new approach to simplifying polygonal 620 and linear features using superpixel segmentation. International Journal of 621 Geographical Information Science, 32(10): 2023-2054.

622 Zhang, M., Yao, W., Meng, L., 2014. Enrichment of topographic road database for the 623 purpose of routing and navigation. International Journal of Digital Earth, 7(5): $411-431$.

625 Zhang, M., 2009. Methods and implementations of road-network matching. Munich: 626 Technical University of Munich 